Phase modulation in high power optical systems caused by pulsed laser-driven particle ablation events

M. J. Matthews, N. N. Shen, A. M. Rubenchik, J. Honig, J. D. Bude

November 21, 2013

SPIE Laser Damage 2013 Boulder, CO, United States September 22, 2013 through September 25, 2013 
This document was prepared as an account of work sponsored by an agency of the United States government. Neither the United States government nor Lawrence Livermore National Security, LLC, nor any of their employees makes any warranty, expressed or implied, or assumes any legal liability or responsibility for the accuracy, completeness, or usefulness of any information, apparatus, product, or process disclosed, or represents that its use would not infringe privately owned rights. Reference herein to any specific commercial product, process, or service by trade name, trademark, manufacturer, or otherwise does not necessarily constitute or imply its endorsement, recommendation, or favoring by the United States government or Lawrence Livermore National Security, LLC. The views and opinions of authors expressed herein do not necessarily state or reflect those of the United States government or Lawrence Livermore National Security, LLC, and shall not be used for advertising or product endorsement purposes. 


\title{
Phase modulation in high power optical systems caused by pulsed laser-driven particle ablation events
}

\author{
Manyalibo J. Matthews*, Nan Shen, Alexander M. Rubenchik, John Honig and Jeff D. Bude \\ Lawrence Livermore National Laboratory, Livermore, CA 94550, USA
}

\begin{abstract}
Surface modification of fused silica windows caused by the laser ablation of surface-bound microparticles is investigated. Using optical and electron microscopies between laser pulses, we detail the ablation, fragmentation and dispersal of 2-150 $\mu \mathrm{m}$ diameter particles of various materials. Following complete ablation and ejection of all debris material, surface pitting was found to be highly dependent on material type and particle size. Subsequent light propagation modeling based on pit morphology indicates up to $\sim 4 \mathrm{x}$ intensification. Understanding this class of non-local, debris-generated damage is argued to be important for effective design of high-power optical windows and debris-mitigation strategies.
\end{abstract}

Keywords: Fourier optics, damage, surfaces, lasers

\section{INTRODUCTION}

Contamination of surfaces is known to be a performance-limiting issue in many areas of technology from microelectronics, bioengineering and optics. ${ }^{1}$ While debris accumulation on optical surfaces is generally problematic because of light scattering, diffraction and obscuration, the impact to performance takes on new dimensions when considering optics for high power laser systems. In these cases, contamination on surfaces generated through optical processing and handling can lead to damage initiation and local fracture that, if left unchecked, can often doom an optic in a pulsed laser system after several successive laser shots. Much attention therefore has been given to studying the effect of contaminating particle material, substrate material, laser energy and pulse length effects which can influence damage initiation. For example, at low enough laser fluences and for a given set of material properties, particle ejection devoid of surface damage has been observed, and in fact exploited for pulsed dry laser cleaning of surfaces. ${ }^{2}$ On the other hand, pulsed laser cleaning of loosely-bound debris on both transmission and reflective optics can be achieved at low pulse energies and has been exploited for various materials. Due to the asymmetry in plasma generation on either surface, damage initiation into the optic surface and laser cleaning tends to be more likely on the exit surface. For this reason, more attention has been given to exit surface contaminant-driven damage initiation. Moreover, little attention has been given to particle-surface energy coupling (Fig. 1) and the degree of melting/pitting following a laser cleaning event compared with the more classic fracture-type damage. While the contaminant may have been removed in this case, the shallow features in the surface that remain can lead to various levels of propagated intensity modulation due to incident phase modulation which in turn may lead to damage. It should be noted that surface modulation effects from contaminants involve not only single shot events in pulsed laser systems, but can require multiple laser shots to materialize as a non-local damage mechanism. A better understanding of such contamination-derived phase objects is therefore warranted to identify under what laser, material and geometric conditions laser damage becomes likely.

In this article we study the morphological evolution of particles of several different materials placed on the entrance surface of an AR-coated fused silica test optic under successive $351 \mathrm{~nm}$ pulsed laser irradiation. Each laser shot heated up the particle to the point of plasma generation, melting and/or detonation which in turn led to dispersal of particle material across the surface. However, the overall behavior depended highly on material absorptivity and thermal properties. For the metals studied (aluminum and stainless steel) absorbed laser energy caused melt flow characterized by hydrodynamic instability. The behavior of the polymer materials (acetal homopolymer- Delrin ${ }^{\mathrm{TM}}$ and polyethylene terephthalate copolymer - PET-G) appeared to depend largely on optical absorptivity at $351 \mathrm{~nm}$ which affected the distribution of heating and subsequent dispersal. The brittle ceramics studied (fused silica and NG-3 absorbing glass) which both had somewhat low absorptivity but higher melting points tended to fracture and 


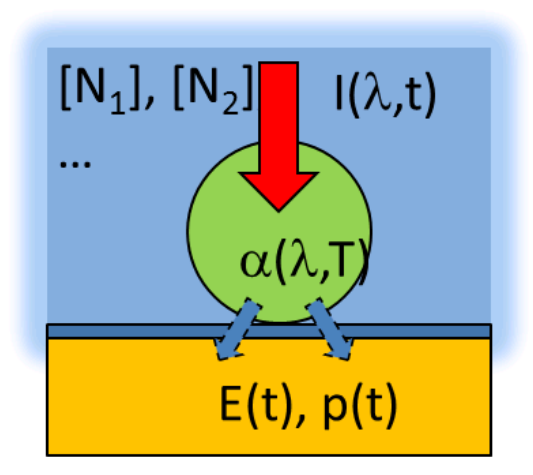

Figure 1. Conceptual picture of laser-particle coupling indicating the interplay of material properties " $\alpha(\lambda, T)$ " laser intensity $\mathrm{I}(\lambda, \mathrm{T})$, gas environment $\left[\mathrm{N}_{\mathrm{i}}\right]$ as they influence energy transfer $\mathrm{E}(\mathrm{t})$ and pressure $\mathrm{p}(\mathrm{t})$ as function of time.

eject from the surface causing minor AR coating removal and minimal surface pitting. On the other hand, aluminum, SS316 and Delrin ${ }^{\mathrm{TM}}$ produced pitting into the fused silica substrate, which was quantified using white light interferometry. The pitting is believed to be caused by evaporation, shock densification and solarization as evidenced by mode shift observed in FTIR microscopy. Wave propagation simulations were performed based on surface height information and used to estimate the probability of damage on the exit surface of the test optic. These predictions were compared with observations of damage initiation on the exit surfaces.

\section{EXPERIMENTAL DETAILS}

Polished UV-grade Corning 7980 fused silica samples were used in the present study, $10 \mathrm{~mm}$ thick by $51 \mathrm{~mm}$ diameter round. Samples were sol gel anti-reflectance coated $(71 \mathrm{~nm}$ thickness $)$ for $351 \mathrm{~nm}$ transmission. A schematic of the sample preparation procedure is shown in Fig. 2. With the exception of silica and pure Al, particles of various sizes were created by using a cleaned, hardened steel file on the materials of interest. These filed particles were captured directly into a stack of two precision sieves. The sieve ranges were $38 \mu \mathrm{m}$ and $55 \mu \mathrm{m}$. The silica particles were generated using $351 \mathrm{~nm}$ laser ablation and captured directly onto a silica test window. The remaining particle materials were deposited onto separate fused silica substrate samples from a height of approximately $30 \mathrm{~cm}$. The substrates were then taken, rotated 90 degrees such that the substrate surface was parallel to the gravity vector, and mechanically tapped to remove the larger and looser particles resulting in a final surface density of $\sim 1$ particle $/ \mathrm{mm}^{2}$. Additional details on the particle sizes are described in Ref. 1.

Following $\sim 1-2$ days of settling time in a clean room environment, samples were loaded individually into a $351 \mathrm{~nm}$ laser damage test chamber described previously. ${ }^{3}$ Briefly, a $27 \mathrm{~mm}$ diameter, flat-top laser beam with $15-20 \%$ beam contrast (ratio of standard deviation to mean) was used with the particles arranged on the incident surface of the sample. Following a $\sim 1 \mathrm{mTorr}$ pump down, all experiments were performed in 2.5 torr of Ar gas. Pulses were shaped using a programmable front end seed pulse system resulting in a $10 \mathrm{~ns}, 0.2 \mathrm{GW} / \mathrm{cm}^{2}$ pre-pulse immediately followed by a $2.5 \mathrm{~ns}, 2.2 \mathrm{GW} / \mathrm{cm}^{2}$ shock pulse. This shape was chosen to roughly approximate an ICF pulse shape used on NIF. ${ }^{4}$ Local irradiance could be determined using a near field camera registered to a CW illuminated transmission camera. In addition, one cycle of loading and unloading the sample into the laser test chamber was performed, without firing the laser, to help ensure that particles that may have been ejected by laser shots were not mistaken for those that fell off unintentionally during sample handling.

Prior to and following each laser shot, each sample was taken out of the laser test chamber, loaded into an SEM chamber (Hitachi, S3400) and a randomly chosen set of particles were imaged and tracked over the course of the experiment. Because the samples could not be $\mathrm{Au}$ or $\mathrm{C}$ coated, some charging was observed. In some cases (e.g. NG3, PET-G), particle remnants were too faint to observe in the SEM past the first laser shot. In other cases, clear, stable remnants could be observed throughout the experiment. In addition to selectively chosen sites imaged with a SEM, the entire sample surface irradiated by the laser was mapped before and after each laser shot using an automated optical microscope. After the completion of the laser testing, samples were characterized using interferometry and IR spectroscopy. Surface height maps were measured using a Zygo New View 100 white light interferometric microscope with $0.5 \mu \mathrm{m}$ lateral and $1 \mathrm{~nm}$ axial spatial resolution. Final volume changes caused by 
particle absorption events were evaluated numerically. Fourier transform infrared (FTIR) spectroscopy was used to evaluate the amount of residual particle content and thermal effect on underlying silica through evaluation of the fictive temperature. Spatially resolved $(\sim 10 \mu \mathrm{m}$ spot size $)$ normal incidence IR reflectance scans of laser-treated regions over the same spectral range were made using a coherent synchrotron radiation source (Advanced Light Source, Lawrence Berkeley National Laboratory) coupled via $15 \mathrm{x} / 0.58 \mathrm{NA}$ reflective optics to a Spectra Tech NicPlan IR microscope. The synchrotron based FTIR (SR-FTIR) measurements were accurate to $0.09 \mathrm{~cm}^{-1}$ with a resolution of $4 \mathrm{~cm}^{-1}$. A lateral step size of $10 \mu \mathrm{m}$ was used to measure variations in IR reflectance as a function of local thermal treatment. Additional details of the SR-FTIR system are described elsewhere. ${ }^{5}$

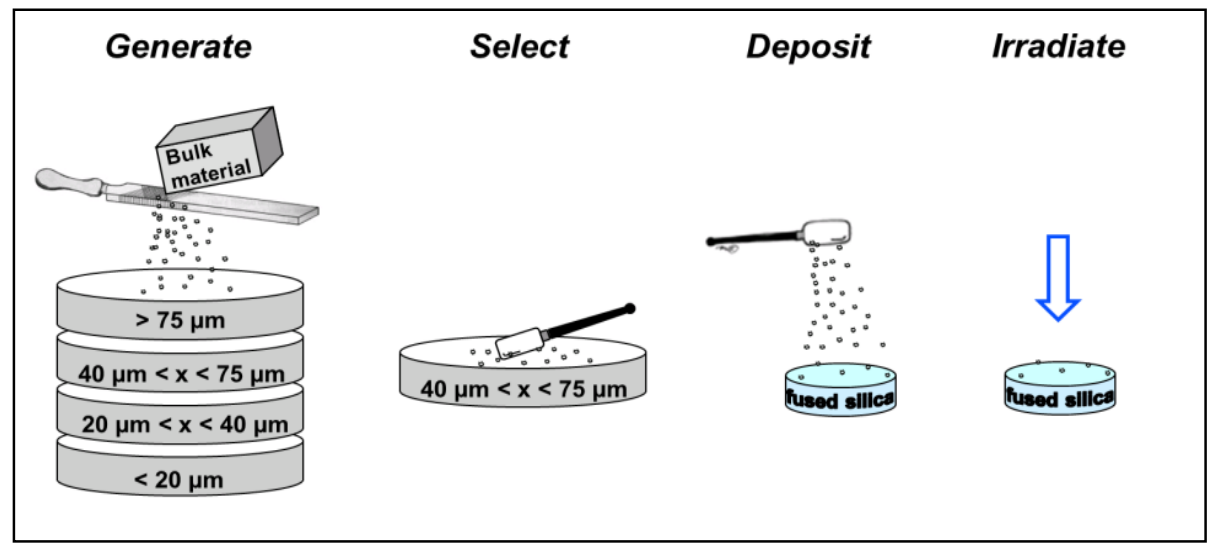

Figure 2. Schematic showing the process used for sample preparation.

\section{RESULTS}

In order to study the energy coupling and effect of laser absorption of the initial laser pulse, a set of particles was studied using SEM. The physical response and dispersal of a typical PET-G particle following the initial laser pulse is shown in Fig. 3. Generally, the polymer particles displayed a similar range of lateral dispersal, but generally the Delrin $^{\mathrm{TM}}$ particles spread less finely than the PET-G. Correspondingly, more material remained localized and available for subsequent laser shot heating in the Delrin ${ }^{\mathrm{TM}}$ case. The morphology of the remaining Delrin ${ }^{\mathrm{TM}}$ material bore some resemblance to that of the silica and SuperGrey particles, but in addition to the break-up of material, the glass particles produced noticeable pitting in the AR coating. These pits, emitting outward from the initial particle position appear as scratches caused by tangentially ejected material. For both glass types, the amount of material remaining localized was roughly the same. In contrast, the metallic particles (aluminum and stainless steel) tended to disperse as liquid with clear evidence of 'splashing' across the surface and droplet formation. Both metals following the initial laser shot - depending on size and local fluence - also contained a more solid-like central core. Generally, the degree of liquid-like morphology of the metallic particles scaled with increasing pulse energy and decreasing particle size. For the more spherical metallic particles, a central low region was observed, possibly indicating capillary effects wherein the momentum of the dispersed liquid carries the central region partially outward producing a donut-shaped distribution.

Following the initial laser shot and surface imaging, each sample was shot until no visible change was observed in the particle debris patterns. The number of shots necessary therefore varied depending on the material. For example, the glass particle - partly owing to the fact that only relatively small particles could be made to adhere initially - samples were shot three times with no visible change after the second shot. On the other hand, six laser exposures were required for debris to disappear or stabilize in the polymer samples, and up to 12 for the metal particle samples. An example of the multi-pulse morphological evolution of the particles is shown in Figure/Video 4 for an aluminum particle. The video associated with Fig. 4 extends over five laser pulses. While only the Delrin $^{\mathrm{TM}}$ and PET-G cases are shown, the behavior of the aluminum and steel particles roughly followed that of the Delrin ${ }^{\mathrm{TM}}$ particles, while the glass particles roughly behaved somewhat like the PET-G. That is, significant material remnants confined radially to $\sim 3 \mathrm{x}$ the initial particle radius remained after the first and second laser pulses for the 


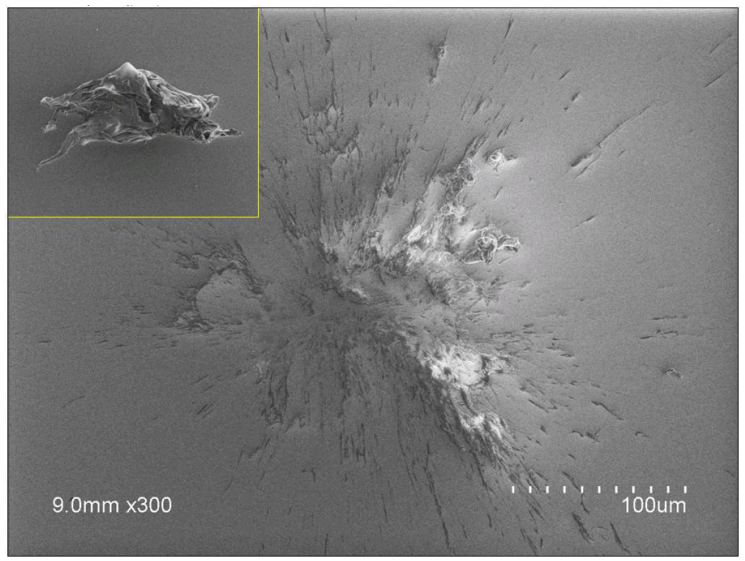

Figure 3. Scanning electron micrograph showing the morphological change in a $\sim 60 \mu \mathrm{m}$ particle of PET-G (inset) following a $9 \mathrm{~J} / \mathrm{cm}^{2}$ laser pulse. Both inset and main figure have the same length scale.

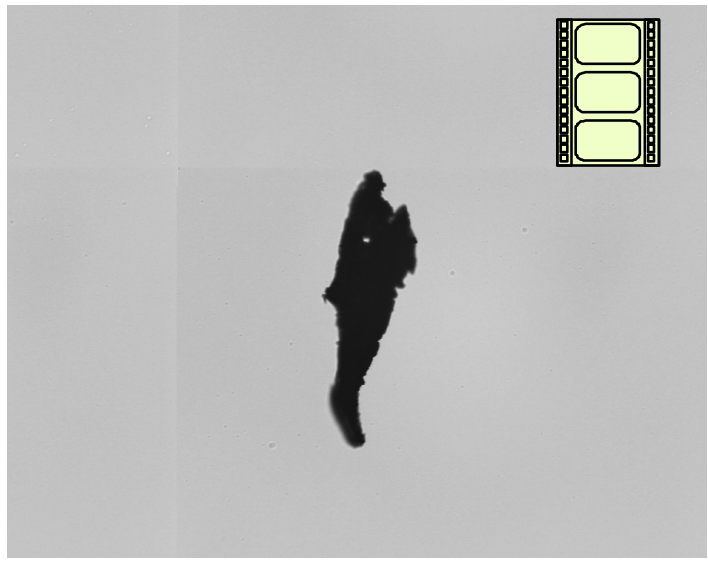

Figure 4, Video 1. (File: Al video 1.gif) Video showing the evolution of a $370 \mu \mathrm{m}$ long $(135 \mu \mathrm{m} \mathrm{ECD)} \mathrm{Al} \mathrm{particle}$ over 5 laser shot sequence.

aluminum, steel and Delrin ${ }^{\mathrm{TM}}$ particles. For PET-G, virtually all material was ejected farther than $3 \mathrm{x}$ the initial particle radius on the initial pulse, with little remaining after the glass particles roughly behaved like the PET-G. That is, significant material remnants confined radially to $\sim 3 \mathrm{x}$ the initial particle radius remained after the first and second laser pulses for aluminum, steel and Delrin ${ }^{\mathrm{TM}}$ particles. For PET-G, virtually all material was ejected farther than $3 x$ the initial particle radius on the initial pulse, with little remaining after the second pulse. The glass particles behaved similar to the PET-G particles in terms of dispersal, though some remnant fractured material persisted in some cases. The PET-G (and glass) related pits contrasted those from the metals in that they tended to produce a pit with a minimum near the center location of the initial particle. On the other hand, a characteristic peaked region in the center of the metal-derived pit (see discussion below) could be related to the general morphology of the initial spreading of material in the first shot as shown in Fig. 3.

The pit volume and depths were evaluated numerically for a range of initial particle diameters as displayed in Fig. 5 . With increasing initial particle area, the final pit volume increased nearly linearly with a slope ranging from $\sim 2.5 \mu \mathrm{m}$ for Al to $\sim 0.06 \mu \mathrm{m}$ for SuperGrey and silica particles. The latter value is consistent with the $71 \mathrm{~nm}$ thickness of the AR coating. However, the maximum pit depth varied considerably and was observed to exceed the coating thickness for most of the cases studied. Interestingly, the two polymers exhibited the most extreme behavior with the PET-G depths limited to $\sim 100 \mathrm{~nm}$ up to initial particle diameters of $130 \mu \mathrm{m}$, while Delrin ${ }^{\mathrm{TM}}$ particles less than $40 \mu \mathrm{m}$ created pit depths in excess of $550 \mathrm{~nm}$. In terms of particle diameter dependence, Fig. 5 displays two ranges of volume production from particle ablation, one in which the volume produced is efficient (diameters less than $\sim 30$ to $\sim 50 \mu \mathrm{m}$ ) and one where the volume dependence on initial diameter is weaker (greater than $50 \mu \mathrm{m})$. This roll-over in behavior could be associated with the break-up of particles on the initial laser shot which tended to disperse material far from the original particle location. Thus, the subsequent pulses ablated less material than would be 
otherwise present. Note that this apparent transition in behavior occurs at smaller diameters for the PET-G and silica particles as compared to the metal particles with the steel transitioning earlier as a function of particle diameter.

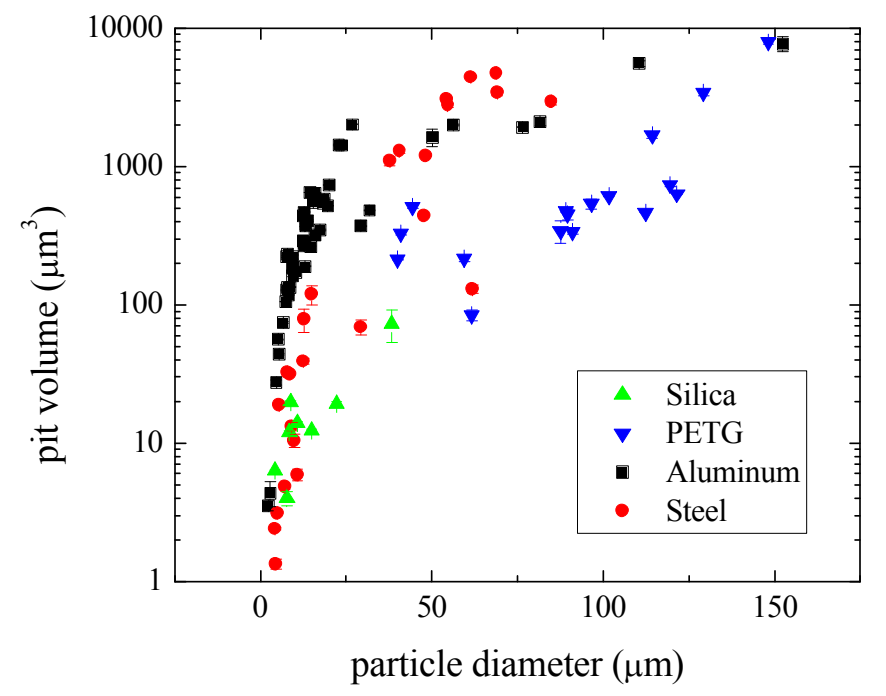

Figure 5. Volume associated with surface pitting caused by multiple laser ablation events as a function of initial particle area-averaged diameter. The error bars represent the range of minimum and maximum background levels used as offsets in the height measurements.

Fourier optical theory was used to describe the propagation and interaction of incident light associated with lasergenerated surface features. Diffraction due to localized surface pitting and depressions can lead to light intensification, which can be characterized by simulating the effective phase imprinted on the optic surface. Following the completion of laser exposure series for each material, individual particle sites were sampled using white light interferometry (Zygo New View 100) with $\sim 500 \mathrm{~nm}$ lateral resolution and $\sim 1 \mathrm{~nm}$ axial resolution. The height maps were then used to calculate phase maps assuming an index change of $-(n-1)=-0.46$ going from air to glass. A $1024 \times 1024$ pixel grid with $2 \mu \mathrm{m} /$ pixel resolution was used to construct a $1.5 \times 1.5 \mathrm{~mm}$ square, $12^{\text {th }}$ order super-Gaussian complex field with unity intensity, to which the calculated phase maps were added. Generally the intensification properties of the final sites followed closely the behavior in pit volumes. That is, the metal particles tended to produce higher contrast intensification patterns, while the glass and polymer (with the exception of Delrin $^{\mathrm{TM}}$ ) tended to produce less pronounced patterns and lower intensification.

Finally, to investigate the microstructural changes caused by the laser ablation events, synchrotron-based IR reflectivity was measured for a set of the sites and compared with spatial variations in surface pitting. Shown in Fig. 6 is a typical case for a $\sim 50 \mu \mathrm{m}$ stainless steel particle subjected to three laser pulses. The interferometry measurements of the profile [Fig. 6(a)] details a 100-200 $\mu \mathrm{m}$ deep pit along with smaller surrounding pits caused by dispersed material being subsequently ablated away. The grey points in Fig. 6(a) correspond to drop-outs from the measurement at locations were remnant steel material was present. These locations are also visible in the reflected (visible) light image in the inset of Fig. 6(a), along with regions where the AR coating has been removed (light grey region roughly $200 \mu \mathrm{m}$ in diameter). This outer coating removed region sits outside a central $\sim 150 \mu \mathrm{m}$ diameter region where the underlying substrate has been affected. Fictive temperature $\left(\mathrm{T}_{\mathrm{F}}\right)$ measurements using vibrational spectroscopy can be used to evaluate the relaxation state, temperature history and density of glasses. ${ }^{6}$ For example, as-received Corning 7980 which has been sufficiently annealed will possess a fictive temperature of $\sim 1315 \mathrm{~K}$. In contrast, sol-gel will tend to have higher fictive temperatures, as well as silica that has been heated and cooled rapidly or shock compressed. Methods to extract $\mathrm{T}_{\mathrm{F}}$ from the measured IR spectrum of silica are described elsewhere. ${ }^{7}$ Figure 6(b) displays the fictive temperature map corresponding to the profile shown in Fig. 6(a). As can be observed, the region away from the ablated region with intact AR coating shows a slightly higher $\mathrm{T}_{\mathrm{F}}(\sim 1400$ $\mathrm{K}$ ) as expected, while the outer coating removed region reveals a $\sim 1300 \mathrm{~K} \mathrm{~T}_{\mathrm{F}}$ region indicative of the underlying Corning 7980 glass. Within this region is a slightly higher $\mathrm{T}_{\mathrm{F}}$ zone which corresponds to the deeper pit regions. 

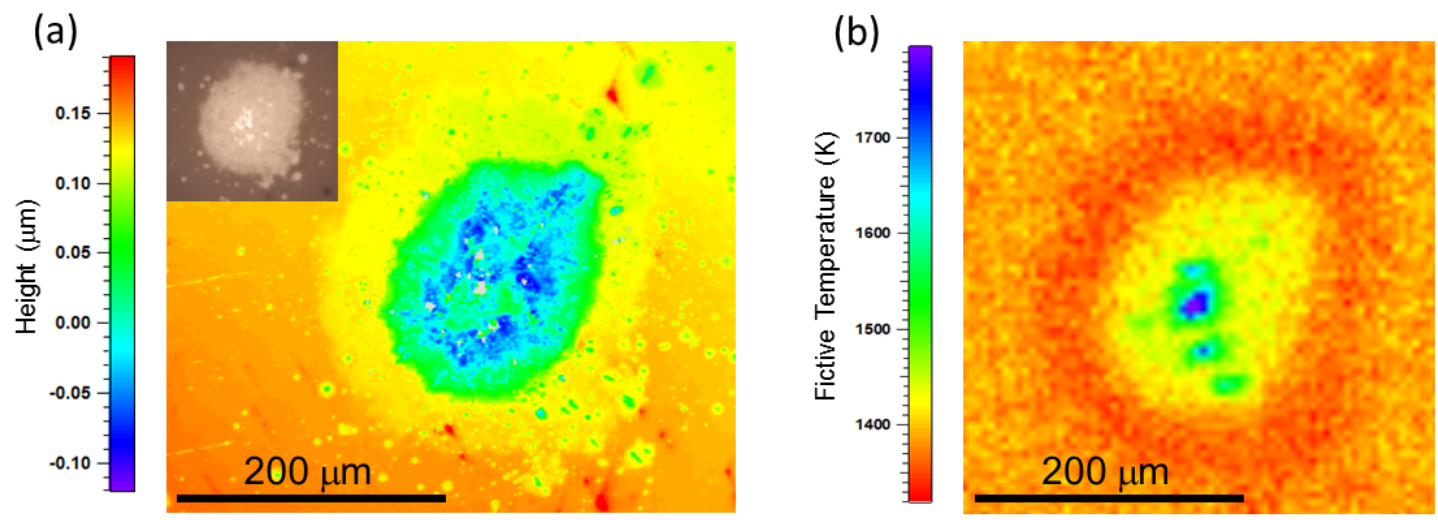

Figure 6. Comparison of the measured surface height profile (a) and the fictive temperature following 3 laser pulses incident on a $\sim 50 \mu \mathrm{m}$ steel particle. The inset of (a) shows the associated reflected light microscope image, indicating the region where the AR coating was removed (lighter region).

\section{DISCUSSION}

The energy coupling and mechanics of particle fragmentation and debris dispersal was found to be highly material dependent. Interestingly, despite the very similar chemistry, physical and thermal properties of Delrin ${ }^{\mathrm{TM}}$ and PET$\mathrm{G}$, these two materials were found to behave very differently in our experiments. Broadly, the initial dispersal, resulting final pit morphology and intensity of simulated light propagation fields was primarily dependent on particle size and opacity. The absorption of light for the metal particles is limited to a depth of $\sim 10 \mathrm{~nm}$, while for the polymers and glasses (in principle) the absorption depths are in excess of $2 \mathrm{~mm}$. However, absorption by defects and scattering (especially for the Delrin ${ }^{\mathrm{TM}}$ ) along the surfaces of these otherwise $351 \mathrm{~nm}$ transparent materials causes inevitable absorption, heating and plasma generation at the surface or bulk of the particles. Rapid temperature and pressure increase then leads to dynamic fracture, yield and phase transitions followed by violent fragmentation and ejection of material across the surface.

A simple model for dynamic fragmentation of a brittle or ductile object under intense impulse (bulk) loading can be argued based on energy balance. ${ }^{8}$ In analogy to Griffith brittle fracture theory, a balance between interfacial (surface) energy and fragment kinetic energy leads to an equilibrium brittle fragment size of

$$
L_{b}=\left[\frac{K_{I c} \sqrt{20}}{\rho c \dot{\epsilon}}\right]^{2 / 3}
$$

where $K_{I c}$ is the fracture toughness, $\rho$ the density, $c$ the velocity of sound and $\dot{\epsilon}$ the linear strain rate. In Eq. (4) the fragment shape is assumed to be spherical and because of the large forces presumed in particle destruction, the contribution from stored elastic energy has been ignored. If we take $\dot{\epsilon}$ to be roughly equal to the inverse pulse length $(\sim 10 \mathrm{~ns})$, and material properties for glass $\left(K_{I c} \sim 0.75 \mathrm{MPa} \sqrt{\mathrm{m}}, \rho \sim 2.2 \mathrm{~g} / \mathrm{cm}^{3}, c \sim 4 \mathrm{~km} / \mathrm{s}\right)$, we find that $L_{b}=2.3$ $\mu \mathrm{m}$, in good agreement with the fragment sizes observed. This fragment size would seem to be a reasonable estimate for the polymer particles, although mechanically one would expect a high degree of plastic yield and flow under both high pressure and significant heating. For a given yield strength $Y$ and critical void fraction $\varepsilon_{c}(\sim 0.1)$, an energy-limited ductile spall process will produce fragments of size $L_{d}$, given by ${ }^{8}$

$$
L_{d}=\left[\frac{8 Y \varepsilon_{c}}{\rho \epsilon^{2}}\right]^{1 / 2}
$$

Again taking $\dot{\epsilon}=1 / \tau$, and properties of Delrin ${ }^{\text {TM }}$ or PET-G at room temperature (Y 53 MPa, $\rho \sim 1.3 \mathrm{~g} / \mathrm{cm}^{3}$ ), one finds a fragment size of about $0.2 \mu \mathrm{m}$, close to the feature size of the PET-G debris field in Fig. 3, but smaller than the larger features observed in other polymers implying that the latter fragmentation may involve combined ductile and brittle behavior. However, because of the low melting points of the polymer particles $\left(450 \mathrm{~K}\right.$ for Delrin ${ }^{\mathrm{TM}}$ and 
$353 \mathrm{~K}$ for PET-G), frictional heating caused by the plasma/vapor recoil pressures likely leads to a mixed phase and liquid flow, despite the long thermal diffusion times across the particles. Specifically, the thermal diffusion constant, $D$, for both homopolymer-type (Delrin ${ }^{\mathrm{TM}}$ ) and PET-G are $\sim 2 \times 10^{-3} \mathrm{~cm}^{2} / \mathrm{s}$ yielding thermal diffusion times $\tau_{t h}=d^{2} / 4 D \sim 3 \mathrm{~ms}$ for $\mathrm{d}=50 \mu \mathrm{m}$ particles. Moreover, because of a slightly higher melting point, but a higher coefficient of thermal expansion than PET-G (150 ppm for Delrin ${ }^{\mathrm{TM}}$ as compared to $68 \mathrm{ppm}$ for PET-G), one may in fact expect to observe more brittle behavior for homopolymer material, which in turn leads to less fluid-like spreading of material and more potency in terms of laser absorption on subsequent laser pulses.
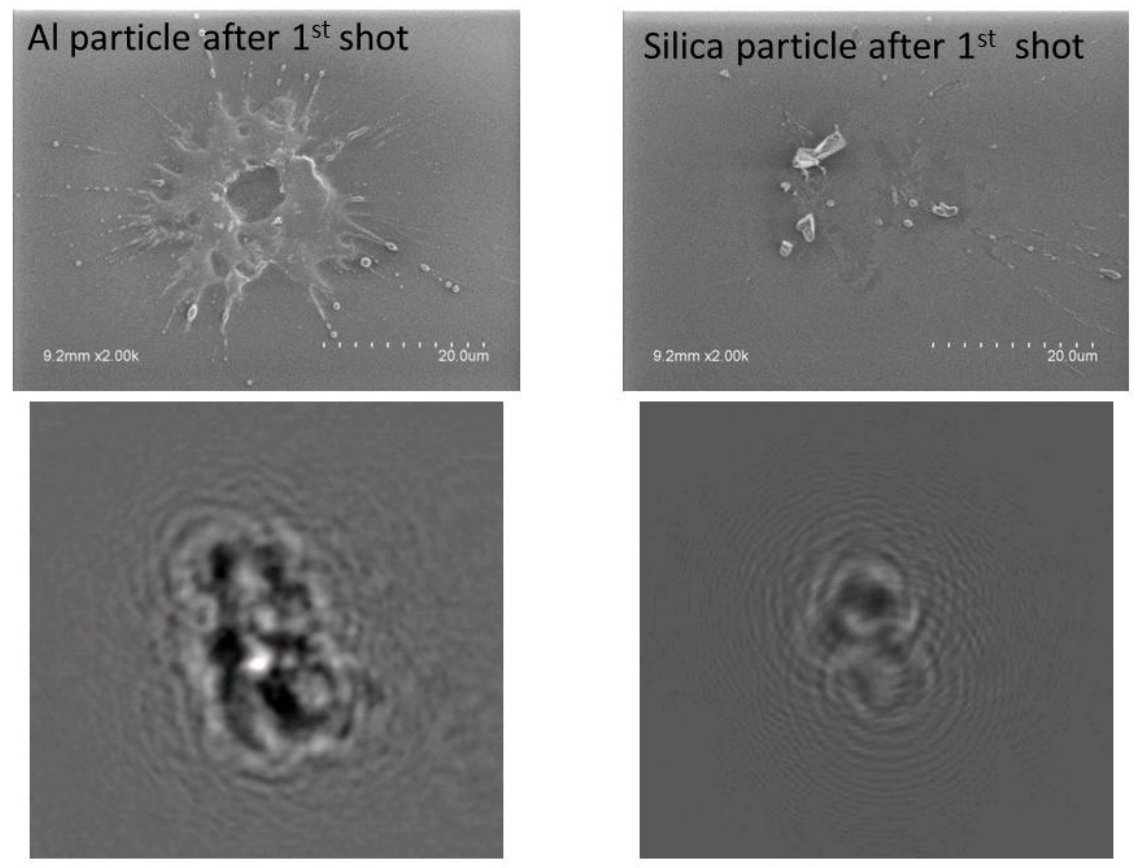

Figure 7. Comparison of initial material dispersal for aluminum and silica (top) and the associated downstream modulation effects calculated from final morphology profiles (bottom). The profiles used in the bottom intensity profiles are representative of the general behavior but do not correspond 1:1 with the particles imaged by SEM in the top of the figure. Note the depression in the aluminum profile at the top that results (after multiple pulses and ablative removal of material) in the hot spot in the intensity profile in the bottom of the figure.

Aluminum and steel particles following surface heating and plasma formation experience large plasma-vapor pressures which tend to laterally extend material outward, but in a more confined area than for more brittle particles due to surface tension effects. The liquid state observed is likely due to frictional melting associated with pressureinduced strain fields. As the material temperature reaches $>10,000 \mathrm{~K}$, internal pressures of $>20 \mathrm{GPa}$ are generated. ${ }^{9}$ Hence, an elastic wave (or shock wave) will propagate to the silica surface on the order of several GPa. When this wave impinges on the silica surface, it can densify the underlying material and is a likely source of the pitting found in these experiments and similar to the shock densification of silica observed from 3 ns laser damage events. ${ }^{5,10}$ At pressures $>7 \mathrm{GPa}$, higher pressure phases of silica are expected, ${ }^{11}$ though from our FTIR analysis detected no discernible variation in the Si bond coordination that would support this. However, the small increase in $\mathrm{T}_{\mathrm{F}}$ shown in Fig. 6(b) is consistent with a high temperature ablation event, and possible compaction due to plasma pressure. Unfortunately, some remnant steel material could affect the IR spectrum in a way that could make precise $T_{F}$ determination difficult. Finally, we note that at high plasma temperatures $(\sim 20 \mathrm{eV})$, it is also possible that soft $\mathrm{x}$-ray and deep ultraviolet light is generated at sufficient intensities to also induce densification. ${ }^{12}$ The liquid like motion of the metal particles tended to produce a 'moat' like structure which reflected the distinctive liquid like dispersal as shown in Fig. 7. Accordingly, the propagated $351 \mathrm{~nm}$ light pattern observed for metal particles tended to have a hot spot at the center aligned with the high point in the surface profile. In contrast, the glass and PET-G particles tended to disperse more randomly and cause less pitting which in turn led to less focusing of downstream propagated light. A complete description of these effects and calculation of the field intensities can be found in Ref. 1. 


\section{CONCLUSIONS}

Surface pitting on silica optical windows created by pulsed laser ablation of micro-particle contaminants and their effect on phase modulation of propagated light was investigated. The potency of the metal particles in producing $>100 \mathrm{~nm}$ pits and light-focusing phase objects was related to their ability to remain absorbing, ductile and localized throughout a sequence of laser pulses. Transparent particles (e.g. PET-G) tended to fragment and disperse widely following the initial laser pulse, consistent with theoretical fragment size estimates, leaving little material to absorb and affect the surface morphology on subsequent laser pulses. Furthermore, the relatively high melting point of the glasses also means that high stresses can build up and cause brittle fracture before the glass can flow and yield under plasma pressure. In contrast, plasma pressures up to $\sim 1 \mathrm{GPa}$ were estimated for the opaque materials, driving ductile yielding and frictional melting which in turn leads to lateral expansion of material after the initial laser pulse. Subsequent laser pulses are believed to cause shock-induced densification, which determines the optical depth of the resulting phase objects. An interesting correlation between debris dispersal, pitting and potential to create a focusing phase object was discussed. This preliminary study could help elucidate the material-dependent behavior affecting ablation-generated phase objects optical systems.

\section{Acknowledgements}

The authors wish to thank W. Gourdin and L. Allison for supplying portions of the particle materials and helpful discussions. H. Bechtel of Lawrence Berkeley National Laboratory is acknowledged for assistance with FTIR measurements on Beamline 5.4 of the Advanced Light Source. We are also grateful to D. Cross, R. Luthi, J. Prior and J. Vickers for sample metrology and laser diagnostics. This work was performed under the auspices of the U.S. Department of Energy by Lawrence Livermore National Laboratory under Contract DE-AC52-07NA27344. LLNLPROC-646621

\section{REFERENCES}

1. M. J. Matthews, N. Shen, J. Honig, J. D. Bude and A. M. Rubenchik, Journal of the Optical Society of America B 30 (12), 3233-3242 (2013).

2. W. Zapka, W. Ziemlich and A. C. Tam, Appl. Phys. Lett. 58 (20), 2217-2219 (1991).

3. M. C. Nostrand, T. L. Weiland, R. L. Luthi, J. L. Vickers, W. D. Sell, J. A. Stanley, J. Honig, J. Auerbach, R. P. Hackel and P. J. Wegner, SPIE 5273, 325-333 (2003).

4. C. A. Haynam, P. J. Wegner, J. M. Auerbach, M. W. Bowers, S. N. Dixit, G. V. Erbert, G. M. Heestand, M. A. Henesian, M. R. Hermann, K. S. Jancaitis, K. R. Manes, C. D. Marshall, N. C. Mehta, J. Menapace, E. Moses, J. R. Murray, M. C. Nostrand, C. D. Orth, R. Patterson, R. A. Sacks, M. J. Shaw, M. Spaeth, S. B. Sutton, W. H. Williams, C. C. Widmayer, R. K. White, S. T. Yang and B. M. Van Wonterghem, Appl. Opt. 46 (16), 3276-3303 (2007).

5. M. J. Matthews, C. W. Carr, H. A. Bechtel and R. N. Raman, Appl. Phys. Lett. 99 (15), 151109 (2011).

6. M. J. Matthews, R. M. Vignes, D. Cooke, S. T. Yang and J. S. Stolken, Opt. Lett. 35 (8), 1311-1313 (2010).

7. M. Tomozawa, J. W. Hong and S. R. Ryu, J. Non-Cryst. Solids 351 (12-13), 1054-1060 (2005).

8. $\quad$ D. E. Grady, J Mech Phys Solids 36 (3), 353-\& (1988).

9. J. C. Crowhurst, M. R. Armstrong, K. B. Knight, J. M. Zaug and E. M. Behymer, Phys. Rev. Lett. 107 (14), $1443021-$ 1443025 (2011).

10. J. Wong, J. L. Ferriera, E. F. Lindsey, D. L. Haupt, I. D. Hutcheon and J. H. Kinney, J. Non-Cryst. Solids 352 (3), $255-$ $272(2006)$.

11. A. Salleo, S. T. Taylor, M. C. Martin, W. R. Panero, R. Jeanloz, T. Sands and F. Y. Genin, Nat. Mater. 2 (12), 796-800 (2003).

12. N. F. Borrelli, C. Smith, D. C. Allan and T. P. Seward, J. Opt. Soc. Am. B-Opt. Phys. 14 (7), 1606-1615 (1997). 